\title{
Cervical lordotic alignment following posterior spinal fusion for adolescent idiopathic scoliosis: reciprocal changes and risk factors for malalignment
}

\author{
Kazunori Hayashi, MD, Hiromitsu Toyoda, MD, PhD, Hidetomi Terai, MD, PhD, \\ Akinobu Suzuki, MD, PhD, Masatoshi Hoshino, MD, PhD, Koji Tamai, MD, Shoichiro Ohyama, MD, \\ and Hiroaki Nakamura, MD, PhD \\ Department of Orthopedic Surgery, Osaka City University Graduate School of Medicine, Osaka, Japan
}

\begin{abstract}
OBJECTIVE Numerous reports have been published on the effectiveness and safety of correction of the coronal Cobb angle and thoracolumbar sagittal alignment in patients with adolescent idiopathic scoliosis (AIS). Suboptimal sagittal alignment, such as decreased thoracic kyphosis (TK), after corrective surgery, is a possible cause of lumbar or cervical spinal degeneration and junctional malalignment; however, few reports are available on reciprocal changes outside of the fused segments, such as the cervical lordotic angle (CLA). This study aimed to investigate the relationship between the perioperative CLA and other radiographic factors or clinical results in AIS, and to identify independent risk factors of postoperative cervical hyperkyphosis.
\end{abstract}

METHODS A total of 51 AIS patients who underwent posterior spinal fusion with the placement of pedicle screw (PS) constructs at thoracic levels were included in the study. Clinical and radiographic follow-up of patients was conducted for a minimum of 2 years, and the postoperative course was evaluated. The authors measured and identified the changes in the CLA and other radiographic parameters using whole-spine radiography, with the patient in the standing position, performed immediately before surgery, 2 weeks after surgery, and 2 years after surgery. The postoperative cervical hyperkyphosis group included patients whose CLA at 2-year follow-up was smaller than $-10^{\circ}$. The reciprocal changes of the CLA and other parameters were also investigated. Univariate and multivariate analyses were conducted to determine the associated risk factors for postoperative cervical hyperkyphosis.

RESULTS This study comprised 48 females and 3 males (mean age 16.0 years). The mean follow-up period was 47 months (range 24-90 months). The main coronal thoracic curve was corrected from $54.6^{\circ}$ to $16.4^{\circ}$, and the mean correction rate was $69.8 \%$ at 2 years. The CLA significantly increased from the mean preoperative measurement $\left(-5.4^{\circ} \pm\right.$ $\left.14^{\circ}\right)$ to the 2-year follow-up measurement $\left(-1.7^{\circ} \pm 11^{\circ}\right)(p=0.019)$. Twelve of the 51 patients had postoperative cervical hyperkyphosis. This group exhibited significantly smaller preoperative CLA and TK measurements $(p=0.001$ and 0.004 , respectively) than the others. After adjusting for confounding factors, preoperative CLA less than $-5^{\circ}$ and preoperative TK less than $10^{\circ}$ were significantly associated with postoperative cervical hyperkyphosis $(p<0.05$; OR 12.5 and 8.59, respectively). However, no differences were found in the clinical results regardless of cervical hyperkyphosis.

CONCLUSIONS The CLA increased significantly from preoperatively to 2 years after surgery. Preoperative small CLA and TK measurements were independent risk factors of postoperative cervical hyperkyphosis. However, there was no difference in the clinical outcomes regardless of cervical hyperkyphosis.

https://thejns.org/doi/abs/10.3171/2016.9.PEDS16298

KEY WORDS cervical lordosis; thoracic kyphosis; spinal fusion; idiopathic scoliosis; risk factors; spine

$\mathrm{P}$ OSTERIOR spinal fusion is a common treatment method for adolescent idiopathic scoliosis (AIS) when conservative treatment is ineffective. Numerous reports have been published on the effectiveness and safety of correction of the coronal Cobb angle and thoracolumbar sagittal alignment. ${ }^{4,13,16}$
Suboptimal sagittal alignment, such as decreased thoracic kyphosis (TK), after corrective surgery is a possible cause of lumbar or cervical spinal degeneration and junctional malalignment. ${ }^{15}$ The kinematic relationship between the global sagittal alignment and cervical parameters, such as the cervical lordotic angle (CLA), in patients with AIS

ABBREVIATIONS AIS = adolescent idiopathic scoliosis; CLA = cervical lordotic angle; LL = lumbar lordosis; PS = pedicle screw; SRS-22r = Scoliosis Research Society22r Patient Questionnaire; SVA = sagittal vertical axis; TK = thoracolumbar kyphosis.

SUBMITTED May 24, 2016. ACCEPTED September 2, 2016.

INCLUDE WHEN CITING Published online January 27, 2017; DOI: 10.3171/2016.9.PEDS16298. 
is not clearly elucidated because of the lack of reports on reciprocal changes outside of the fused segments after posterior corrective spinal fusion. ${ }^{8,9,12,18}$ There are only a few radiographic and clinical studies that evaluate both preoperative and postoperative courses of patients with cervical hyperkyphosis. Some studies have identified risk factors for postoperative cervical hyperkyphosis after AIS surgery. However, none of the previous studies performed multivariate analyses that would control for potential interactions in an attempt to identify independent risk factors., ${ }^{9,12}$

In this study, we examined pre- and postoperative CLAs, as well as other radiographic parameters and clinical outcomes of AIS patients. Factors associated with postoperative cervical hyperkyphosis in univariate and multivariate analyses were also identified. We hypothesized that perioperative CLA is an indicator for an important relationship between TK and the sagittal vertical axis (SVA), which may be independent risk factors for postoperative cervical malalignment. Furthermore, height distraction at surgery or height growth at the 2-year follow-up may also be potential risk factors. Postoperative cervical hyperkyphosis may predict suboptimal clinical results.

\section{Methods \\ Patient Population}

A total of 54 consecutive patients with AIS who underwent posterior corrective fusion at thoracic levels with the placement pedicle screw (PS) constructs at our institution from 2008 to 2013 were enrolled in the study. Among these patients, 51 had a minimum follow-up of 2 years and underwent complete radiographic and clinical evaluation. Informed consent was obtained from all individual participants and guardians, and the institutional review board of the Osaka City Graduate School of Medicine approved this study. Patients who required anterior fusion surgery, those who had previous spine surgery, and/or those who had a history of neuromuscular diseases were excluded from the study. The patients were divided into 3 groups. Group A (cervical hyperkyphosis group) included patients whose CLA at the 2-year follow-up was smaller than $-10^{\circ}$. Patients whose CLA ranged from $-10^{\circ}$ to $0^{\circ}$ were included in Group B (normal cervical kyphotic curve group). Group C (cervical lordotic group) included patients whose CLA was larger than $0^{\circ}$. Radiographs of a typical case from each group are shown in Fig. 1.

\section{Surgical Treatment}

Three surgeons performed posterior corrective fusion in the same manner as previously described. ${ }^{21}$ After muscle exposure to the costotransverse joint, the transverse process above T-10 and the inferior articular processes at all fused levels were bilaterally resected. Segmental/multilevel PSs were inserted into the vertebrae with or without the aid of a CT-based navigation system (StealthStation S7, Medtronic Sofamor Danek). A transverse hook system was used only on the upper instrumented vertebra. The mean implant density was 1.55 in this cohort. A prebent $6.35-\mathrm{mm}$ pure titanium rod was first set on the convex side and then rotated approximately $90^{\circ}$ by pushing the patient's hump (convex manipulation methods). After pre- fixed coronal and sagittal bending, in situ contouring was conducted under careful monitoring of the spinal motor evoked potentials. A prebent $6.35-\mathrm{mm}$ titanium alloy rod was introduced on the concave side, and direct vertebral rotation was then performed in the desired vertebrae, particularly around the apex level. Two rods were finally fixed with 2 cross-links. Final adjustments were made under fluoroscopy with additional in situ contouring and compression-distraction. Two instrumentation systems were used for corrective surgery. The CD HORIZON LEGACY 6.35 Spinal System (Medtronic Sofamor Danek) was used in 24 cases, while the EXPEDIUM 6.35 Spine System (DePuy Synthes Spine) was used in the remaining 27 cases.

\section{Clinical Outcomes and Radiographic Evaluations}

Clinical outcomes were evaluated for each domain of the Scoliosis Research Society-22r Patient Questionnaire (SRS-22r) administered 2 years postsurgery in each group. ${ }^{1}$ The domains measured consisted of physical/activity, pain, self-image, and mental health, and mean subtotal and total scores were calculated. ${ }^{1}$ In addition, surgery-related and potentially relevant complications caused by the cervical spine, as well as reoperations, were identified. Full-length standing anteroposterior and lateral radiographs were obtained in a standardized upright position. Lateral-view whole-spine radiographs were obtained with the patients in upright, relaxed, and "hands on clavicle" positions. The CLA (C2-7) was determined as the angle formed by the line along the posterior body of $\mathrm{C}-2$ and the line along the posterior body of C-7. Thoracic kyphosis (TK; T5-12) was defined as the Cobb angle between the cranial endplate of T-5 and the caudal endplate of T-12. Lumbar lordosis (LL; L1-5) was defined by as angle between the cranial endplates of L-1 and S-1. The C-7 sagittal vertical axis (SVA) was defined as the horizontal distance from the superior posterior end of the upper sacral endplate to the C-7 or C-2 plumb lines. The CLA, TK, LL, and SVA were measured immediately before surgery, 2 weeks after surgery, and 2 years after surgery. Each measurement is shown in Fig. 2. Two independent spine surgeons (K.H. and H.T.) performed the radiographic measurements; the mean values of the measurements were then used for further analyses. The kappa coefficient of interrater reliability for CLA preoperatively, at 2 weeks postoperatively, and at 2 years postoperatively was $0.98,0.95$, and 0.90 , respectively. We investigated the postoperative course in Group A and compared it with that of the other 2 groups.

\section{Analysis of Associated Risk Factors for Postoperative Cervical Hyperkyphosis}

From medical records and operative charts, we recorded patient demographics, duration of the operation, blood loss, number of fused levels, number of PSs, height distraction at surgery (height at 1 week after surgery - preoperative height), height growth at 2 years postoperatively, the apex of the main curve, Lenke type and sagittal modifiers (hypokyphosis [-], normal kyphotic curve [N], and hyperkyphosis [+]), Cobb angle of the coronal main curve, and postoperative correction rates, as well as other coro- 


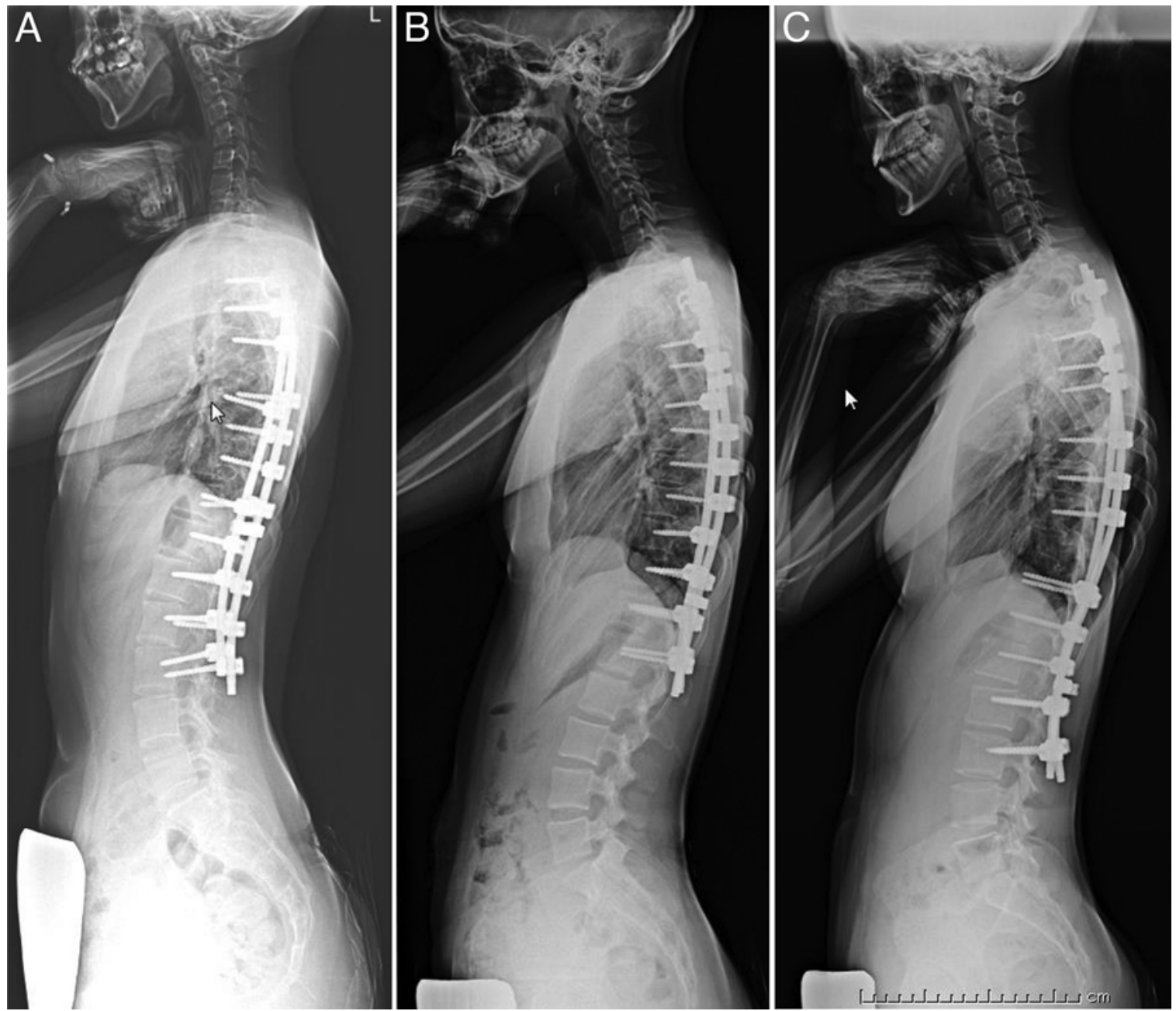

FIG. 1. Whole-spine radiographs of typical cases: Group A (A), Group B (B), and Group C (C).

nal and sagittal radiographic parameters taken pre- and postoperatively. Associated risk factors of postoperative cervical hyperkyphosis were also evaluated.

\section{Statistical Analysis}

Statistical correlations were determined using Welch modification 1-way ANOVA, Games-Howell's multiple comparison for continuous valuables, and the chi-square test for categorical variables in univariate analysis; $\mathrm{p}<$ 0.05 was considered statistically significant. Multivariate logistic regression analysis was used to adjust for potential confounding factors, and odds ratios with $95 \%$ confidence intervals were calculated. The factors included using forced entry methods into the multivariate model were those with $\mathrm{p}<0.10$ in univariate analysis. All statistical analyses were conducted using SPSS (version 19.0, IBM).

\section{Results}

\section{Patient Data and Reciprocal Change in CLA}

The patient population comprised 48 girls and 3 boys. The mean follow-up period was 47 months (range 24-90 months). All patients were Asian (50 were Japanese). The mean age at surgery was 16.0 years, and the mean body mass index was 19.0. A total of 37 cases were classified into Lenke Type 1, 7 cases were Type 2, and 7 cases were Type $3 .{ }^{14}$ Likewise, 13 cases were classified into the Lenke sagittal modifier "-", 35 cases into "N", and 3 cases into "+." For surgical parameters, the mean number of fused levels was 10.5; the upper and lower instrumented vertebral distributions are presented in Table 1 . The main coronal thoracic curve was corrected from $54.6^{\circ}$ to $16.4^{\circ}$, and the mean correction rate was $69.8 \%$ at 2 years. Height distraction at surgery was $1.9 \mathrm{~cm}$ on average, and $2.7-\mathrm{cm}$ distraction and growth was observed at 2 years postoperatively from the preoperative period. The CLA significantly increased from $-5.4^{\circ} \pm 14^{\circ}$ preoperatively to $1.0 \pm$ $12^{\circ}$ at 2 weeks postoperatively and then decreased to $-1.7^{\circ}$ $\pm 11^{\circ}$ at 2 years postoperatively $(\mathrm{p}=0.019)$. The SVA significantly increased from $4.9 \pm 32 \mathrm{~mm}$ preoperatively to $13.5 \pm 37 \mathrm{~mm}$ at 2 weeks postoperatively and decreased to $-10.9 \pm 26 \mathrm{~mm}$ at 2 years postoperatively $(\mathrm{p}=0.002)$. TK increased significantly from $16.0^{\circ} \pm 9.4^{\circ}$ preoperatively to $19.0^{\circ} \pm 6.7^{\circ}$ at 2 weeks postoperatively and further increased to $20.6^{\circ} \pm 6.8^{\circ}$ at 2 years postoperatively ( $\mathrm{p}=$ $0.001)$. 


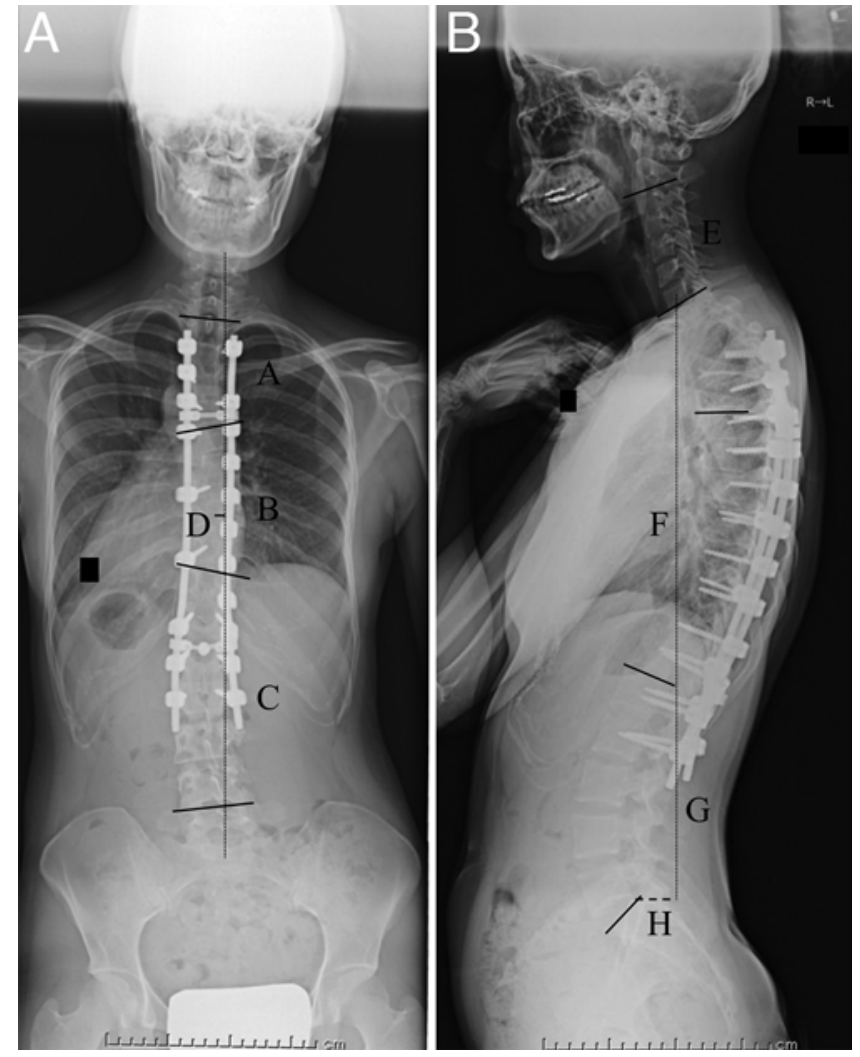

FIG. 2. Measurement methods of each parameter as seen on anteroposterior $(\mathbf{A})$ and lateral $(\mathbf{B})$ radiographs: Cobb angle of main thoracic curve (A), Cobb angle of proximal curve (B), Cobb angle of distal curve (C), apical vertebral translation (D), CLA (E), TK (F), LL (G), and SVA $(H)$.

\section{Postoperative Radiographic Evaluation in Cervical Hyperkyphosis Cases}

There were 12 patients in Group A, 16 in Group B, and 23 in Group C. The mean postoperative CLAs of these cases were $-16.4^{\circ},-4.3^{\circ}$, and $7.8^{\circ}$, respectively. Reciprocal changes in CLA and TK in each group are shown in Fig. 3. CLA significantly increased in all groups at 2 weeks postoperatively; however, it decreased at 2 years postoperatively in Groups A and B. In particular, the CLA in Group A was restored to its original condition at 2 years postoperatively. Meanwhile, the TK of each group stably increased until 2 years postoperatively. Group A exhibited a greater increase in TK after surgery, but achieved the lowest TK values. Patient and radiographic data of the 3 groups are summarized in Table 2. Group A had a significantly smaller preoperative CLA $(p=0.001)$ than Group C. Similarly, Group A had significantly smaller preoperative TK $(p=0.004)$ than Groups B and C. Moreover, Group A had a significantly smaller postoperative TK than Group C ( $p=0.043)$. Neither perioperative coronal parameters nor height changes exhibited significant differences among the 3 groups.

Multivariate analysis revealed that the independent risk factors for postoperative cervical hyperkyphosis (Group A) were preoperative cervical kyphosis with a preoperative CLA less than $-5^{\circ}$ (OR 12.5, 95\% CI 1.63-95.2) and preoperative TK less than $10^{\circ}$ (OR 8.59, 95\% CI 1.35-
TABLE 1. Distribution of the upper and lower instrumented vertebrae

\begin{tabular}{cc}
\hline Vertebra & No. of Patients \\
\hline UIV & \\
\hline T-2 & 13 \\
\hline T-3 & 25 \\
\hline T-4 & 10 \\
\hline T-5 & 2 \\
\hline T-6 & 1 \\
\hline LIV & \\
\hline T-11 & 4 \\
\hline T-12 & 7 \\
\hline L-1 & 10 \\
\hline L-2 & 15 \\
\hline L-3 & 15 \\
\hline
\end{tabular}

LIV = lower instrumented vertebra; UIV = upper instrumented vertebra.
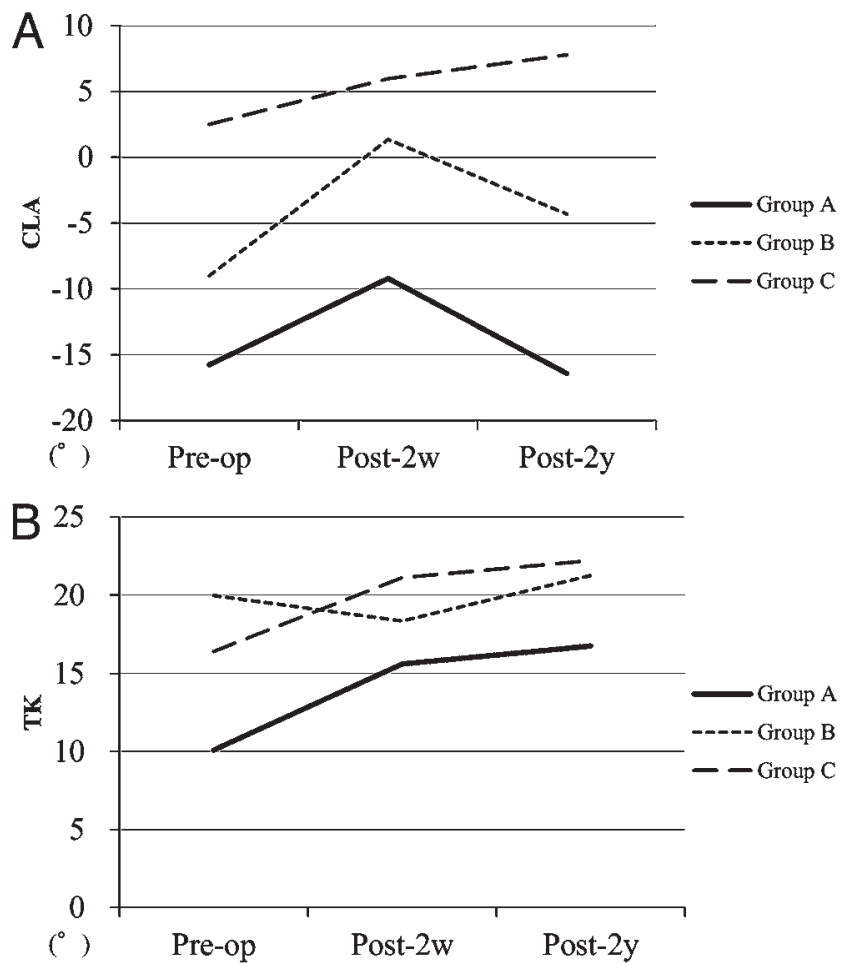

FIG. 3. Line graph displaying the difference in reciprocal change in CLA $(A)$ and TK (B) in each cervical alignment group. Post-2w = 2 weeks postoperatively; post-2y $=2$ years postoperatively.

54.50), without any potential interaction. Hosmer-Lemeshow analysis showed a $p$ value of 0.60 , and Nagelkerke analysis showed an $\mathrm{R}^{2}$ value of 0.41 . TK and SVA at 2 years postoperatively were excluded in this model.

\section{Postoperative Clinical Results in Cervical Hyperkyphosis Patients}

No significant differences between groups in terms of SRS-22r domains (Fig. 4). The number of surgery-related 
TABLE 2. Patient and radiographic data

\begin{tabular}{|c|c|c|c|c|c|c|}
\hline Variable & Total $(n=51)$ & Group A $(n=12)$ & Group B $(n=16)$ & Group C ( $n=23)$ & p Value & Multiple Comparisons* \\
\hline \multicolumn{7}{|l|}{ Patient demographics } \\
\hline Age (yrs) & 16.0 & 16.0 & 15.0 & 16.7 & 0.25 & \\
\hline Sex, no. & & & & & 0.32 & \\
\hline Male & 3 & 0 & 0 & 3 & & \\
\hline Female & 48 & 12 & 16 & 20 & & \\
\hline Risser grade & 4.10 & 4.25 & 4.00 & 4.09 & 0.76 & \\
\hline Height (m) & 1.58 & 1.58 & 1.58 & 1.57 & 0.92 & \\
\hline $\mathrm{BMI}\left(\mathrm{kg} / \mathrm{m}^{2}\right)$ & 19.0 & 18.6 & 19.1 & 19.1 & 0.53 & \\
\hline \multicolumn{7}{|l|}{ Op characteristics } \\
\hline Op time (mins) & 367 & 380 & 345 & 375 & 0.47 & \\
\hline Blood loss (ml) & 1285 & 1479 & 1474 & 1052 & 0.14 & \\
\hline No. of fused levels & 10.5 & 10.8 & 10.5 & 10.3 & 0.44 & \\
\hline No. of PSs & 17.8 & 17.9 & 17.1 & 18.0 & 0.40 & \\
\hline Height distraction after op (cm) & 1.9 & 2.2 & 1.7 & 2.0 & 0.76 & \\
\hline Height growth at 2 yrs (cm) & 2.8 & 2.8 & 2.8 & 2.7 & 0.99 & \\
\hline \multicolumn{7}{|l|}{ Scoliosis characteristics } \\
\hline Lenke type, no. & & & & & 0.21 & \\
\hline 1 & 37 & 6 & 12 & 19 & & \\
\hline 2 & 7 & 4 & 2 & 1 & & \\
\hline 3 & 7 & 2 & 2 & 3 & & \\
\hline Lenke sagittal modifiers, no. & & & & & 0.39 & \\
\hline- & 13 & 5 & 2 & 6 & & \\
\hline $\mathrm{N}$ & 35 & 6 & 13 & 16 & & \\
\hline+ & 3 & 1 & 1 & 1 & & \\
\hline Apex of curve, no. & & & & & 0.27 & \\
\hline $\mathrm{T}-7$ & 5 & 1 & 3 & 1 & & \\
\hline $\mathrm{T} 7 / 8-\mathrm{T} 8 / 9$ & 23 & 8 & 6 & 9 & & \\
\hline T9-11 & 23 & 3 & 7 & 13 & & \\
\hline \multicolumn{7}{|l|}{ Preop characteristics } \\
\hline Main curve $\left(^{\circ}\right)$ & 54.6 & 51.5 & 55.1 & 55.9 & 0.25 & \\
\hline Proximal curve $\left(^{\circ}\right)$ & 28.5 & 29.6 & 30.0 & 26.9 & 0.57 & \\
\hline Distal curve $\left({ }^{\circ}\right)$ & 33.4 & 34.7 & 31.6 & 34.0 & 0.78 & \\
\hline Apical vertebral translation (mm) & 44.6 & 43.0 & 44.3 & 45.7 & 0.81 & \\
\hline $\operatorname{CLA}\left({ }^{\circ}\right)$ & -5.4 & -15.8 & -9.0 & 2.5 & 0.001 & bł, cf \\
\hline $\mathrm{TK}\left({ }^{\circ}\right)$ & 16.0 & 10.1 & 20.0 & 16.4 & 0.004 & ał, c† \\
\hline $\operatorname{LL}\left({ }^{\circ}\right)$ & 44.7 & 44.2 & 47.6 & 42.8 & 0.44 & \\
\hline SVA (mm) & 4.9 & 7.8 & -0.3 & 6.9 & 0.65 & \\
\hline \multicolumn{7}{|l|}{ 2-yr postop characteristics } \\
\hline Main curve $\left({ }^{\circ}\right)$ & 16.4 & 18.2 & 14.3 & 17.0 & 0.24 & \\
\hline Correction rate (\%) & 69.8 & 64.3 & 74.0 & 69.8 & 0.17 & \\
\hline Proximal curve $\left(^{\circ}\right)$ & 16.3 & 16.3 & 16.6 & 16.2 & 0.99 & \\
\hline Distal curve $\left({ }^{\circ}\right)$ & 12.1 & 13.0 & 11.0 & 12.4 & 0.70 & \\
\hline Apical vertebral translation (mm) & 10.1 & 13.4 & 8.6 & 9.5 & 0.55 & \\
\hline $\operatorname{CLA}\left({ }^{\circ}\right)$ & -1.7 & -16.4 & -4.3 & 7.8 & $<0.001$ & $a \neq, b \neq, c f$ \\
\hline $\mathrm{TK}\left({ }^{\circ}\right)$ & 20.6 & 16.8 & 21.3 & 22.2 & 0.043 & c† \\
\hline $\operatorname{LL}\left({ }^{\circ}\right)$ & 48.0 & 47.5 & 50.7 & 46.3 & 0.22 & \\
\hline SVA (mm) & -10.9 & -25.4 & -8.9 & -4.6 & 0.14 & \\
\hline
\end{tabular}

$\mathrm{BMI}=$ body mass index.

Values are the means unless stated otherwise

* $a$, Group A vs B; b, Group B vs C; and c, Group A vs C.

$\dagger p<0.01$

$\ddagger p<0.05$. 


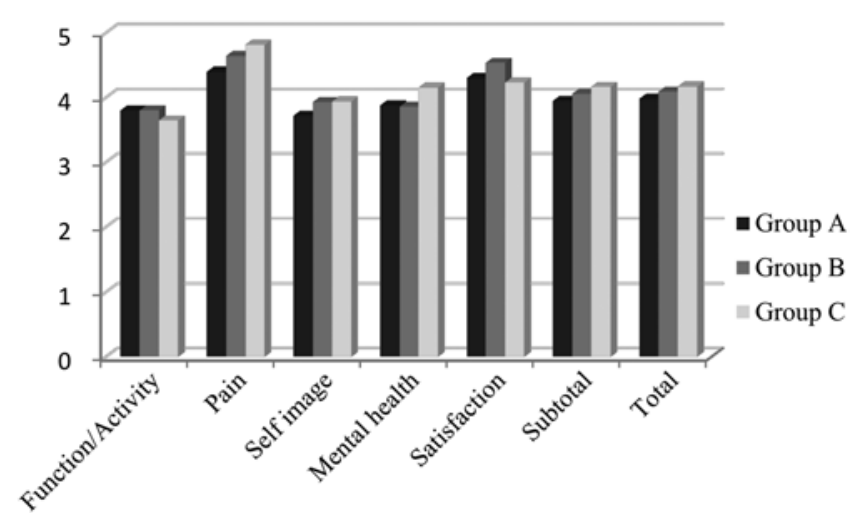

FIG. 4. Bar graph showing the absence of a significant difference in SRS-22r scores between the groups.

or potentially relevant complications caused by cervical spine, as well as reoperation cases, is summarized in Table 3 .

\section{Discussion}

The CLA significantly increased from preoperatively $\left(-5.4^{\circ} \pm 14^{\circ}\right)$ to 2 years after posterior corrective surgery for AIS patients $\left(-1.7^{\circ} \pm 11^{\circ}\right)$. Patients with postoperative cervical kyphosis had a significantly smaller preoperative CLA than patients with cervical lordosis, as well as a smaller preoperative TK than the others. Multivariate analysis revealed that preoperative small CLA and small TK measurements were independent risk factors for postoperative cervical kyphosis. However, postoperative cervical kyphosis patients did not have poorer clinical outcomes than the others. To our knowledge, this study is the first to report independent risk factors for postoperative cervical kyphosis after posterior corrective fusion for AIS.

Radiographic data of cervical sagittal alignment in healthy populations have been reported..$^{11,17,23}$ A CLA in the 10 - to 20 -year-old healthy individuals ranges from $0^{\circ}$ to $10^{\circ}$. A previous study reported that the CLA in AIS patients ranged from $-10^{\circ}$ to approximately $0^{\circ} .^{5-8}$ In the present study, the mean preoperative CLA was $-5.5^{\circ}$, which is in agreement with the aforementioned findings of previous studies.

Cervical alignment is associated with thoracolumbar sagittal alignment in spinal care. Winter et al. ${ }^{22}$ reported that cervical kyphosis in AIS is a compensatory phenomenon accompanied by thoracic lordosis. However, few reports about reciprocal cervical changes after correction surgery of thoracic alignment for scoliosis are available. Hilibrand et al. ${ }^{8}$ first examined the sagittal alignment of the cervical spine in 38 patients with AIS; posterior corrective surgery was performed to treat AIS with CotrelDubousset or Harrington instrumentation. They confirmed a positive correlation between preoperative CLA and TK. In addition, no progression of cervical kyphosis was observed in patients with preoperative "hypo TK," but the CLA in "normal TK" patients significantly increased at 1-year follow-up. Hwang et al. ${ }^{9}$ investigated the postoperative CLA in 22 patients treated with all-PS constructs. They reported that surgical treatment using all-PS con-
TABLE 3. Surgery-related and cervical potentially relevant complications of cervical spine

\begin{tabular}{|c|c|c|c|}
\hline Complication & $\begin{array}{c}\text { Group A } \\
(n=12)\end{array}$ & $\begin{array}{l}\text { Group B } \\
(n=14)\end{array}$ & $\begin{array}{l}\text { Group C } \\
(n=25)\end{array}$ \\
\hline \multicolumn{4}{|l|}{ Surgery related } \\
\hline Instrument failure around UIV & & & 1 \\
\hline Deterioration of proximal curve & & & 1 \\
\hline \multicolumn{4}{|l|}{ Potentially related to cervical spine } \\
\hline Transient extremity numbness & 1 & & \\
\hline Transient lower-extremity numbness & & 1 & \\
\hline Reop & & & 1 \\
\hline
\end{tabular}

structs has a significant hypokyphotic effect on the thoracic sagittal plane alignment and that postoperative TK is excessively decreased; the cervical spine may decompensate into significant kyphosis during the follow-up period. Legarreta et al. ${ }^{12}$ compared 25 cases that were treated using all-PS constructs and Cotrel-Dubousset constructs after 2 years. They noted that the former exhibited more hypokyphotic effects of the thoracic spine with greater decompensation of the cervical spine.

Compared with previous reports, ${ }^{7-9}$ this study found a substantial increase in CLA at the 2-year follow-up. This increase may be attributed to an increase in TK after surgery, while a decrease in TK was reported in previous studies. Presumably, the difference in TK transition depends on the choice of surgical method and the difference in preoperative thoracolumbar alignment. The patients in the present study had relatively smaller TK and LL measurements preoperatively than those in the previous studies. The healthy Japanese population has been reported to have smaller TK and LL measurements than the healthy Caucasian population. ${ }^{6}$ In these circumstances, we had to obtain adequate correction of TK postoperatively; thus, we paid particular attention to sagittal contouring during surgical intervention.

Cervical alignment is strongly influenced by other sagittal parameters. Sugimoto et al. ${ }^{20}$ reported that preoperative cervical alignment correlated with SVA in AIS patients. In regard to postoperative conditions, Hwang et al. ${ }^{9}$ noted differences in CLA reciprocal transition after posterior corrective fusion between patients with cervical hyperkyphosis and those with a normal cervical kyphotic curve preoperatively. Ha et al. ${ }^{7}$ also described the reciprocal relationship between preoperative CLA and postoperative CLA. An increase in TK is reported to be associated with a decrease in CLA $;{ }^{10} \mathrm{LL}$ is a significant correlation parameter with CLA. ${ }^{3}$ However, parameters of the sagittal plane, such as CLA, TK, and LL, are all closely related to each other. Therefore, examining the relationships among multiple variables at the same time is important. Lonner et al. ${ }^{15}$ investigated multiple regression analysis for thoracic alignment. They found that preoperative TK and Cobb angle are independent factors associated with postoperative TK. In the present study, we evaluated the independent risk factors of postoperative cervical hyperkyphosis by using multivariate logistic regression analysis and found that preoperative cervical kyphosis (preoperative CLA $<-5^{\circ}$, 
OR 8.59) and preoperative TK less than $10^{\circ}$ (OR 12.5) were independently associated factors without any potential interaction. With regard to surgical intervention, the fact that patients with small preoperative TK tend to have postoperative cervical hyperkyphosis may indicate that an ideal global postoperative sagittal alignment cannot be obtained in these patients. This is the clinical implication of this study, and spine surgeons must practice care in sagittal alignment during surgical intervention, especially in patients with smaller TK measurements.

Contrary to our hypothesis, neither height distraction at surgery nor height growth at 2 years was a risk factor of cervical kyphosis. Height distraction is due to straightening of scoliosis, and the magnitude of the coronal Cobb correction and the number of fused levels correlated to height gain after posterior corrective fusion..$^{19}$ In this study, neither Cobb correction rates nor the number of fused levels were accompanied by cervical alignment. This result may be a reason for the lack of correlation between cervical alignment and height.

This study has several limitations. First, the sample size was small, but the characteristics of postoperative cervical kyphosis cases and independent risk factors could be studied. Second, this study lacked data on corresponding healthy adolescents, and the follow-up period was short. We could not determine whether cervical kyphosis increases the risk for the future development of neck pain or cervical myelopathy in scoliosis patients. We hypothesized that postoperative cervical kyphosis patients would achieve suboptimal clinical results, because in degenerative conditions, kyphotic cervical alignment leads to worse clinical results. ${ }^{2}$ Clinical assessment through adulthood should be performed in a future study. Third, this study retrospectively analyzed prospectively collected data. Determination of an ideal TK for AIS patients is difficult; therefore, whether surgical treatment for a good alignment of TK offers good alignment of CLA is unclear. Fourth, the cervical alignment established by whole-spine radiography tends to be affected by factors such as patient rotation, posture, and the angle at which the radiograph was obtained. The present study did not completely resolve this problem, which may have created some bias.

\section{Conclusions}

We demonstrated that CLA increased significantly at the 2-year follow-up after posterior corrective fusion for AIS patients. Preoperative cervical kyphosis and preoperative small TK were independent risk factors of postoperative cervical malalignment. However, cervical kyphosis did not affect the clinical results.

\section{Acknowledgments}

English proofreading was performed by Editage.

\section{References}

1. Asher M, Min Lai S, Burton D, Manna B: The reliability and concurrent validity of the Scoliosis Research Society-22 patient questionnaire for idiopathic scoliosis. Spine (Phila Pa 1976) 28:63-69, 2003

2. Batzdorf U, Batzdorff A: Analysis of cervical spine curva- ture in patients with cervical spondylosis. Neurosurgery 22:827-836, 1988

3. Charles YP, Sfeir G, Matter-Parrat V, Sauleau EA, Steib JP: Cervical sagittal alignment in idiopathic scoliosis treated by posterior instrumentation and in situ bending. Spine (Phila Pa 1976) 40:E419-E427, 2015

4. Di Silvestre M, Bakaloudis G, Lolli F, Vommaro F, Martikos K, Parisini P: Posterior fusion only for thoracic adolescent idiopathic scoliosis of more than 80 degrees: pedicle screws versus hybrid instrumentation. Eur Spine J 17:1336-1349, 2008

5. Edgar MA, Mehta MH: Long-term follow-up of fused and unfused idiopathic scoliosis. J Bone Joint Surg Br 70:712716, 1988

6. Endo K, Suzuki H, Nishimura H, Tanaka H, Shishido T, Yamamoto K: Characteristics of sagittal spino-pelvic alignment in Japanese young adults. Asian Spine J 8:599-604, 2014

7. Ha Y, Schwab F, Lafage V, Mundis G, Shaffrey C, Smith J, et al: Reciprocal changes in cervical spine alignment after corrective thoracolumbar deformity surgery. Eur Spine J 23:552-559, 2014

8. Hilibrand AS, Tannenbaum DA, Graziano GP, Loder RT, Hensinger RN: The sagittal alignment of the cervical spine in adolescent idiopathic scoliosis. J Pediatr Orthop 15:627632,1995

9. Hwang SW, Samdani AF, Tantorski M, Cahill P, Nydick J, Fine A, et al: Cervical sagittal plane decompensation after surgery for adolescent idiopathic scoliosis: an effect imparted by postoperative thoracic hypokyphosis. J Neurosurg Spine 15:491-496, 2011

10. Ilharreborde B, Vidal C, Skalli W, Mazda K: Sagittal alignment of the cervical spine in adolescent idiopathic scoliosis treated by posteromedial translation. Eur Spine J 22:330337, 2013

11. Lee CS, Noh H, Lee DH, Hwang CJ, Kim H, Cho SK: Analysis of sagittal spinal alignment in 181 asymptomatic children. J Spinal Disord Tech 25:E259-E263, 2012

12. Legarreta CA, Barrios C, Rositto GE, Reviriego JM, Maruenda JI, Escalada MN, et al: Cervical and thoracic sagittal misalignment after surgery for adolescent idiopathic scoliosis: a comparative study of all pedicle screws versus hybrid instrumentation. Spine (Phila Pa 1976) 39:13301337, 2014

13. Lehman RA Jr, Lenke LG, Keeler KA, Kim YJ, Buchowski JM, Cheh G, et al: Operative treatment of adolescent idiopathic scoliosis with posterior pedicle screw-only constructs: minimum three-year follow-up of one hundred fourteen cases. Spine (Phila Pa 1976) 33:1598-1604, 2008

14. Lenke LG, Betz RR, Harms J, Bridwell KH, Clements DH, Lowe TG, et al: Adolescent idiopathic scoliosis: a new classification to determine extent of spinal arthrodesis. J Bone Joint Surg Am 83-A:1169-1181, 2001

15. Lonner BS, Lazar-Antman MA, Sponseller PD, Shah SA, Newton PO, Betz R, et al: Multivariate analysis of factors associated with kyphosis maintenance in adolescent idiopathic scoliosis. Spine (Phila Pa 1976) 37:1297-1302, 2012

16. Mulpuri K, Perdios A, Reilly CW: Evidence-based medicine analysis of all pedicle screw constructs in adolescent idiopathic scoliosis. Spine (Phila Pa 1976) 32 (19 Suppl):S109_ S114, 2007

17. Park MS, Moon SH, Lee HM, Kim SW, Kim TH, Lee SY, et al: The effect of age on cervical sagittal alignment: normative data on 100 asymptomatic subjects. Spine (Phila Pa 1976) 38:E458-E463, 2013

18. Roussouly P, Labelle H, Rouissi J, Bodin A: Pre- and postoperative sagittal balance in idiopathic scoliosis: a comparison over the ages of two cohorts of 132 adolescents and 52 adults. Eur Spine J 22 (Suppl 2):S203-S215, 2013

19. Spencer HT, Gold ME, Karlin LI, Hedequist DJ, Hresko MT: 
Gain in spinal height from surgical correction of idiopathic scoliosis. J Bone Joint Surg Am 96:59-65, 2014

20. Sugimoto Y, Tanaka M, Takigawa T, Tetsunaga T, Mazaki T, Ozaki T: Cervical kyphosis in patients with idiopathic scoliosis. J Spine Res 6:44-48, 2015

21. Terai H, Toyoda H, Suzuki A, Dozono S, Yasuda H, Tamai $\mathrm{K}$, et al: A new corrective technique for adolescent idiopathic scoliosis: convex manipulation using $6.35 \mathrm{~mm}$ diameter pure titanium rod followed by concave fixation using $6.35 \mathrm{~mm}$ diameter titanium alloy. Scoliosis 10 (Suppl 2):S14, 2015

22. Winter RB, Lovell WW, Moe JH: Excessive thoracic lordosis and loss of pulmonary function in patients with idiopathic scoliosis. J Bone Joint Surg Am 57:972-977, 1975

23. Yukawa Y, Kato F, Suda K, Yamagata M, Ueta T: Age-related changes in osseous anatomy, alignment, and range of motion of the cervical spine. Part I: Radiographic data from over 1,200 asymptomatic subjects. Eur Spine J 21:1492-1498, 2012

\section{Disclosures}

The authors report no conflict of interest concerning the materi- als or methods used in this study or the findings specified in this paper.

\section{Author Contributions}

Conception and design: Hayashi. Acquisition of data: Toyoda, Hayashi. Analysis and interpretation of data: Hayashi. Drafting the article: Hayashi. Critically revising the article: Toyoda, Terai. Reviewed submitted version of manuscript: all authors. Approved the final version of the manuscript on behalf of all authors: Toyoda. Statistical analysis: Hayashi. Study supervision: Toyoda, Terai.

\section{Supplemental Information}

Previous Presentations

The results of this study were presented at the 44th Annual Meeting of the Japanese Spine Surgery and Related Research in Fukuoka, Japan, April 16-18, 2015.

\section{Correspondence}

Hiromitsu Toyoda, 1-4-3 Asahimachi Abeno-Ku, Osaka 5458585, Japan.email: h-toyoda@msic.med.osaka-cu.ac.jp. 